\title{
Transient ischemia induces massive nuclear accumulation of SUMO2/3-conjugated proteins in spinal cord neurons
}

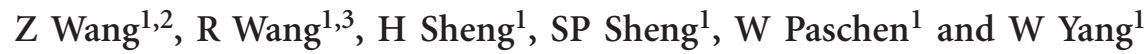

Objectives: The objective of this study is to determine whether transient spinal cord ischemia activates small ubiquitin-like modifier (SUMO1-3) conjugation, a post-translational protein modification that protects neurons from ischemia-like conditions.

Methods: Mice were subjected to 8-12 min of spinal cord ischemia and 3-24 h of recovery using a newly developed experimental model. To characterize the model, activation of stress response pathways induced after spinal cord ischemia, previously observed in other experimental models, was verified by western blot analysis. Levels and subcellular localization of SUMO-conjugated proteins in spinal cords were evaluated by western blot analysis and immunohistochemistry, respectively.

Results: Following transient spinal cord ischemia, stress responses were activated as indicated by increased phosphorylation of eukaryotic initiation factor 2 (elF2 $\alpha$ ), extracellular signal-regulated kinases (ERK1/2) and Akt. SUMO1 conjugation was not altered, but a selective rise in levels of SUMO2/3-conjugated proteins occurred, peaking at $6 \mathrm{~h}$ reperfusion. The marked activation of SUMO2/ 3 conjugation was a neuronal response to ischemia, as indicated by co-localization with the neuronal marker NeuN, and was associated with nuclear accumulation of SUMO2/3-conjugated proteins.

Conclusion: Our study suggests that spinal cord neurons respond to ischemic stress by activation of SUMO2/3 conjugation. Many of the identified SUMO target proteins are transcription factors and other nuclear proteins involved in gene expression and genome stability. It is therefore concluded that the post-ischemic activation of SUMO2/3 conjugation may define the fate of neurons exposed to a transient interruption of blood supply, and that this pathway could be a therapeutic target to increase the resistance of spinal cord neurons to transient ischemia.

Spinal Cord (2013) 51, 139-143; doi:10.1038/sc.2012.100; published online 4 September 2012

Keywords: ischemia; SUMO; neuroprotection; stress response

\section{INTRODUCTION}

Paraplegia resulting from spinal cord ischemia is the most debilitating complication associated with thoracoabdominal aortic aneurysm repair. ${ }^{1}$ Various protective approaches have been proposed to decrease the incidence and severity of spinal cord injury. These include epidural cooling, distal aortic perfusion and cerebrospinal fluid drainage. ${ }^{2-4}$ To establish new approaches to protect the spinal cord from ischemic damage, a better understanding of the various processes triggered by an insufficient blood supply to the spinal cord is required. The most promising strategy would be to activate endogenous neuroprotective pathways before the surgical procedure and thus, increase the resistance of spinal cord neurons to a transient interruption of blood supply. The small ubiquitin-like modifier (SUMO) conjugation pathway could be such a pathway that protects cells from ischemic insults when activated.

SUMO1-3 is a group of small proteins binding to lysine residues of target proteins, thereby modulating their stability, activity and subcellular localization. A large number of SUMO-conjugation target proteins are transcription factors and other nuclear proteins involved in gene expression and genome stability. ${ }^{5,6}$ Any substantial change in levels of SUMO-conjugated proteins is therefore expected to have a major impact on the fate of metabolically stressed cells.
We have shown that the SUMO2/3 conjugation pathway is massively activated after both transient focal and global cerebral ischemia. ${ }^{7,8}$ The pattern of changes in SUMO2/3 conjugation induced by transient focal cerebral ischemia suggests that this is a protective stress response shielding neurons from damage induced by a transient interruption of blood supply. Changes are most pronounced in neurons located at the border of the ischemia tissue where various protective pathways are activated and a short non-lethal duration of vascular occlusion was sufficient to markedly activate this pathway. ${ }^{7}$ However, although cortical neurons located at the border of infarcts exhibit a dramatic post-ischemic activation of SUMO2/3 conjugation, striatal neurons show only minor activation. ${ }^{7}$ This suggests that the SUMO conjugation pathway is not activated in all neurons exposed to a transient interruption of blood supply.

Results from several studies suggest that, in the brain, SUMO conjugation represents an endogenous-protective response in neurons stressed by transient ischemia. Whether spinal cord neurons respond to a transient ischemia by activating the SUMO conjugation pathway has not yet been determined. In this study, we evaluated the effects of transient spinal cord ischemia on SUMO conjugation. We used a recently established mouse model in which the middle segment of the thoracic aorta is cross-clamped via left lateral thoracotomy. ${ }^{9}$

\footnotetext{
${ }^{1}$ Department of Anesthesiology, Multidisciplinary Neuroprotection Laboratories, Duke University Medical Center, Durham, NC, USA; ${ }^{2}$ Department of Neurosurgery, The First Affiliated Hospital, Zhengzhou University, Zhengzhou, China and ${ }^{3}$ Department of Nuclear Medicine, The First Affiliated Hospital, Zhengzhou University, Zhengzhou, China Correspondence: Dr W Yang, Department of Anesthesiology, Multidisciplinary Neuroprotection Laboratories, Duke University Medical Center, 152 Sands Building, Research Drive, Durham, NC 27710, USA.

E-mail: wei.yang@duke.edu

Received 10 May 2012; revised 2 August 2012; accepted 3 August 2012; published online 4 September 2012
} 
Animals were subjected to 8,10 , or 12 min of spinal cord ischemia followed by up to $24 \mathrm{~h}$ of recovery. Following transient spinal cord ischemia, a marked increase in levels and nuclear accumulation of SUMO2/3-conjugated proteins was observed. Considering the neuroprotective effect of $\mathrm{SUMO} 2 / 3$ conjugation, ${ }^{10}$ this pathway could be a new target for therapeutic intervention to increase the resistance of neurons to a transient interruption of blood supply.

\section{MATERIALS AND METHODS}

The Duke University Animal Care and Use Committee approved this study. Male C57Bl/6J mice (8-10 weeks old, 20-25g, Jackson Laboratories, Bar Harbor, ME, USA) were fasted over night with free access to water. Spinal cord ischemia was performed using a newly developed simplified model. ${ }^{9}$ In short, animals were anesthetized with isoflurane, the trachea was orally intubated and both lungs were mechanically ventilated. Inspired isoflurane was set to $1.8 \%$ during surgical preparation and ischemia. Rectal temperature was controlled and maintained at $37.0 \pm 0.5^{\circ} \mathrm{C}$. Mice were placed in the right lateral decubitus position and an incision was made between the eighth and ninth ribs to expose the thoracic aorta. Spinal cord ischemia was induced by placing a small human aneurysm clip on the aorta at the level of T8. At the end of the defined ischemia interval, the clip was removed, the wound was closed and isoflurane was discontinued. Mice were continuously ventilated until spontaneous respiration resumed. The trachea was extubated upon recovery of the righting reflex.

For western blot analysis, animals were anesthetized with isoflurane and euthanized, and spinal cords were excised, dissected and immediately frozen in liquid nitrogen and stored at $-80{ }^{\circ} \mathrm{C}$ until the tissue was processed for analysis. Lumbar segments were analyzed for ischemic changes, whereas T1-T6 segments were used as control. Frozen samples were weighed in the cold, and proteins were extracted using lysis buffer composed of $\beta$-glycerophosphate (50 $\mathrm{mmoll}^{-1}$; pH 7.4), $1 \mathrm{mmoll}^{-1}$ EDTA, $1 \mathrm{mmoll}^{-1}$ EGTA, $0.5 \mathrm{mmoll}^{-1}$ $\mathrm{Na}_{3} \mathrm{VO}_{4}$ and $2 \%$ SDS to block de-SUMOylation of SUMO-conjugated proteins. ${ }^{11}$ To further reduce the risk of de-SUMOylation during protein extraction, frozen samples were added to 10 volumes of lysis buffer and immediately homogenized by a short sonication for $10 \mathrm{~s}$ followed by heating to $95^{\circ} \mathrm{C}$ for $10 \mathrm{~min}$. Protein concentration of extracts was analyzed using the BCA protein assay (Thermo Scientific, Rockford, IL, USA). Immunoblotting was performed using $4-15 \%$ or $10 \%$ SDS-PAGE gels (Bio-Rad, Hercules, CA, USA). Bands were transferred to PVDF membranes (Bio-Rad) and membranes were blocked for $1 \mathrm{~h}$ in Tris-buffered saline solution supplemented with $0.1 \%$ Tween 20 and 5\% skim milk powder, and incubated with the primary antibody for $16 \mathrm{~h}$ at $4{ }^{\circ} \mathrm{C}$. The following primary antibodies were used: rabbit antieukaryotic initiation factor 2 (eIF2 $\alpha$ ) (\#9722 Cell Signaling, Danvers, MA, USA), rabbit anti-phospho eIF2 $\alpha$ (\#9721 Cell Signaling), rabbit anti-extracellular signal-regulated kinases (ERK1/2) (\#4695 Cell Signaling), rabbit antiphospho ERK1/2 (\#4370 Cell Signaling), rabbit anti-Akt (\#4691 Cell Signaling), rabbit anti-phospho Akt (Ser473; \#4060 Cell Signaling) and rabbit antiSUMO1 and anti-SUMO2/3 (dilution 1:1000; by courtesy of Dr John Hallenbeck). Monoclonal antibodies against $\beta$-actin and GAPDH (SigmaAldrich, St Louis, MO, USA) were used as loading control. To quantify levels of SUMO1- and SUMO2/3-conjugated proteins, image analysis was performed using the ImageJ program (Wayne Rasband, NIH, Bethesda, MD, USA). The high-molecular-weight area in each lane, as indicated in Figure $2 \mathrm{~A}-\mathrm{C}$, was cropped and analyzed. For quantification, film background optical density was measured and subtracted from SUMO conjugation optical density. Data are presented as means \pm s.d. (fold increase vs control), with $n=3-5$ independent samples per group. Statistically significant differences between control and experimental groups were evaluated by ANOVA followed by Fisher's protected least significant differences test. A probability of $95 \%$ was taken to indicate significant differences between the groups.

To evaluate changes in SUMO conjugation at the cellular level following ischemia, animals were anesthetized with isoflurane and the lungs were mechanically ventilated. Intra-aortic perfusion was performed using $10 \%$ buffered formalin solution, and the lumbar segment of the spinal cord was dissected, fixed overnight and immersed in $20 \%$ sucrose at $4{ }^{\circ} \mathrm{C}$. Transverse sections $(20 \mu \mathrm{m})$ were cut on a Leica cryostat. Immunofluorescence staining was performed on free-floating sections. After overnight incubation at $4{ }^{\circ} \mathrm{C}$ with a mixture of rabbit anti-SUMO2/3 (1:500) and mouse anti-NeuN (1:500; MAB377, Chemicon, Billerica, MA, USA), sections were incubated with Alexa Fluor 594-conjugated goat anti-rabbit $\operatorname{IgG}$ (1:500; Invitrogen, Carlsbad, CA, USA) and Alexa Fluor 488-conjugated goat anti-mouse IgG (1:500; Invitrogen) at room temperature. DNA was stained with 4,6-diamidino-2-phenylindole in a mounting medium (Santa Cruz Technology, Santa Cruz, CA, USA). Overview of whole sections was generated by stitching together multiple images on an Axio Observer Z1 motorized fluorescence microscope (Carl Zeiss MicroImaging, Jena, Germany). Confocal images were captured on an LSM 510 confocal microscope (Carl Zeiss MicroImaging) using a $\times 40 / 1.3$ oil objective.

We certify that applicable institutional and governmental regulations concerning the ethical use of animals were followed during the course of this research.

\section{RESULTS}

In the present study, we used a simple and reliable new animal model to induce spinal cord ischemia in mice. ${ }^{9}$ To characterize this model in more detail and verify a pattern of stress responses similar to that elicited in other well-established rabbit and rat models of spinal cord ischemia, we evaluated post-ischemic changes in levels of phosphorylation of eIF $2 \alpha,{ }^{12}$ ERK $1 / 2^{13}$ and the serine-threonine kinase Akt. ${ }^{14}$ During reperfusion after $10 \mathrm{~min}$ of ischemia, a marked increase in the levels of p-eIF2 $\alpha$, p-ERK1/2 and p-Akt was evident, whereas the levels of the total proteins did not change (Figure 1). These data confirmed that three major pathways activated in other spinal cord ischemia models are also activated in our new mouse model.

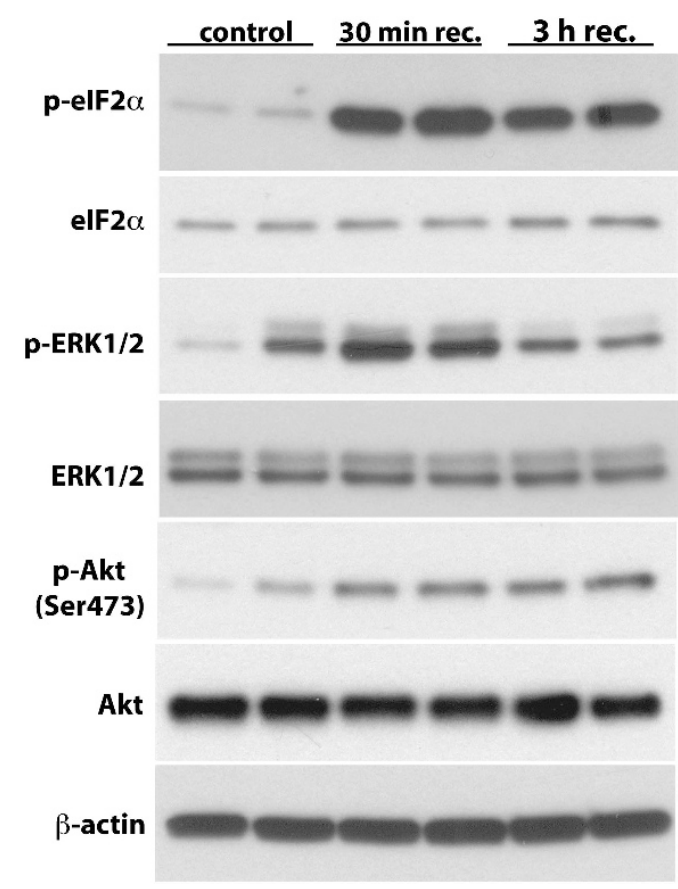

Figure 1 Characterization of the spinal cord ischemia model in mice. Western blot analysis depicts the phosphorylation pattern of elF $2 \alpha$, ERK $1 / 2$ and Akt in the spinal cord of mice (two animals per group) subjected to 10 min of thoracic aorta cross-clamping at the level of T8, and 30 min or $3 \mathrm{~h}$ of recovery (rec.). Lumbar spinal cord segments served as ischemia samples, whereas T1-T6 segments served as controls. Tissue specimens were processed for western blot analysis as described in detail in Materials and methods. A monoclonal antibody against $\beta$-actin was used as loading control. 
A

SUMO1 conjugation in spinal cord ischemia 3h reperfusion
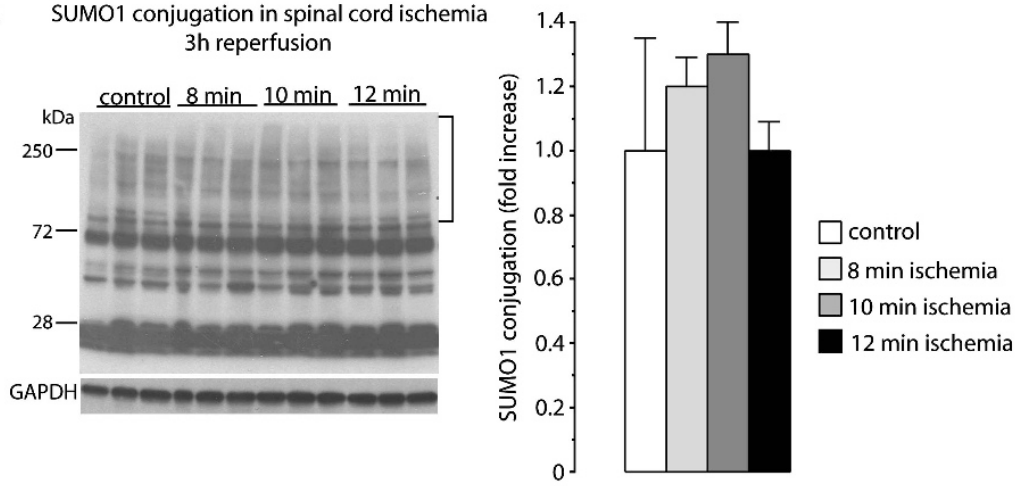

B
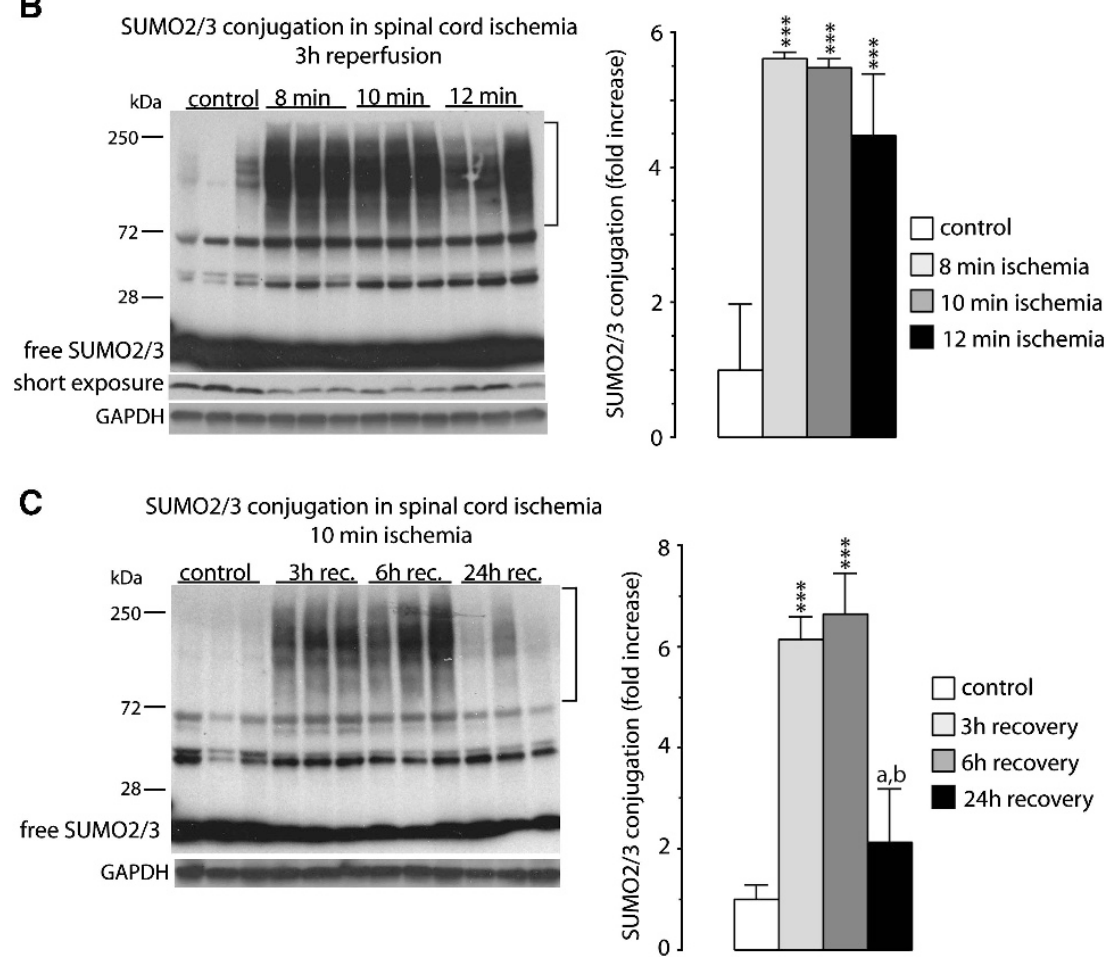

Figure 2 Transient spinal cord ischemia is followed by a marked increase in levels of SUMO2/3-conjugated proteins. Western blot analysis depicts the pattern and summary of SUMO1 (A) and SUMO2/3 (B and C) conjugation in spinal cord samples. Spinal cord samples were taken and processed as described in detail in Materials and methods. Lumbar spinal cord segments served as ischemia samples, whereas T1-T6 segments served as controls. Monoclonal antibody against GAPDH was used as loading control. The high-molecular-weight area in each lane, as indicated in A-C, was cropped and analyzed. Quantification data are presented as means \pm s.d. (fold increase vs control; $n=3-5$ /group). ${ }^{* * *} P<0.001$ (experimental group vs control); ${ }^{\mathrm{a}} \mathrm{P}<0.001$ ( $24 \mathrm{~h}$ vs $3 \mathrm{~h}$ of reperfusion); ${ }^{\mathrm{b}} \mathrm{P}<0.001$ ( $24 \mathrm{~h}$ vs $6 \mathrm{~h}$ of reperfusion; ANOVA, followed by Fisher's PLSD test).

To evaluate changes in SUMO conjugation following transient spinal cord ischemia, animals were subjected to 8,10 or $12 \mathrm{~min}$ of spinal cord ischemia followed by $3 \mathrm{~h}$ of reperfusion, and levels of SUMO1- and SUMO2/3-conjugated proteins were analyzed by western blot. We did not find any major changes in levels of SUMO1-conjugated proteins in spinal cords of animals subjected to $8-12 \mathrm{~min}$ of ischemia and $3 \mathrm{~h}$ of reperfusion (Figure $2 \mathrm{~A}$ ) or in spinal cords of animals subjected to $10 \mathrm{~min}$ of ischemia followed by 3,6 or $24 \mathrm{~h}$ of reperfusion (data not shown).

Levels of SUMO2/3-conjugated proteins were markedly increased at $3 \mathrm{~h}$ of reperfusion: $5.6 \pm 0.09,5.5 \pm 0.14$ and $4.5 \pm 0.91$-fold after 8 , 10 , or $12 \mathrm{~min}$ of ischemia, respectively (Figure $2 \mathrm{~B}$; $P<0.001$ vs control). The rise in levels of SUMO2/3-conjugated proteins was associated with a decrease in levels of free SUMO2/3, from $100 \pm 7 \%$ in control spinal cord segments to $54 \pm 5 \%$ ( $P<0.01$ vs control), $58 \pm 5 \%(P<0.01$ vs control $)$ and $85 \pm 23 \%$ of control after 8,10 , or $12 \mathrm{~min}$ of ischemia and $3 \mathrm{~h}$ of recovery, respectively. The effects of $10 \mathrm{~min}$ of ischemia and 3,6 , or $24 \mathrm{~h}$ of reperfusion on levels of SUMO2/3-conjugated proteins are shown in Figure 2C. Levels of SUMO2/3-conjugated proteins rose $6.1 \pm 0.44(P<0.001$ vs control), $6.6 \pm 0.81(P<0.001$ vs control $)$ and $2.1 \pm 1.06$-fold at 3,6 , and $24 \mathrm{~h}$ of recovery, respectively.

To evaluate post-ischemic changes in levels of SUMO2/3-conjugated proteins at the cellular and subcellular level, SUMO2/3 immunofluorescence staining was performed together with staining for the neuronal marker NeuN (Figure 3). Experimental animals were subjected to $10 \mathrm{~min}$ of spinal cord ischemia and $6 \mathrm{~h}$ of reperfusion. SUMO2/3 immunoreactivity was confined to neurons as identified by 
A
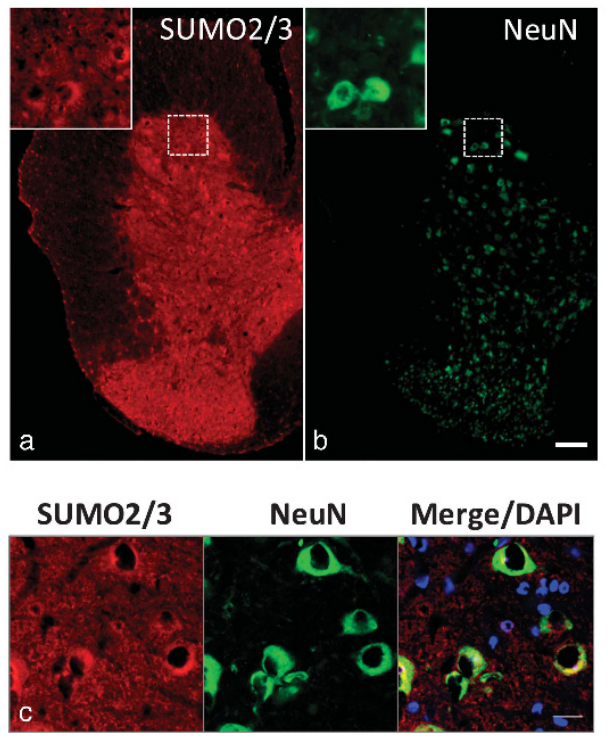

B
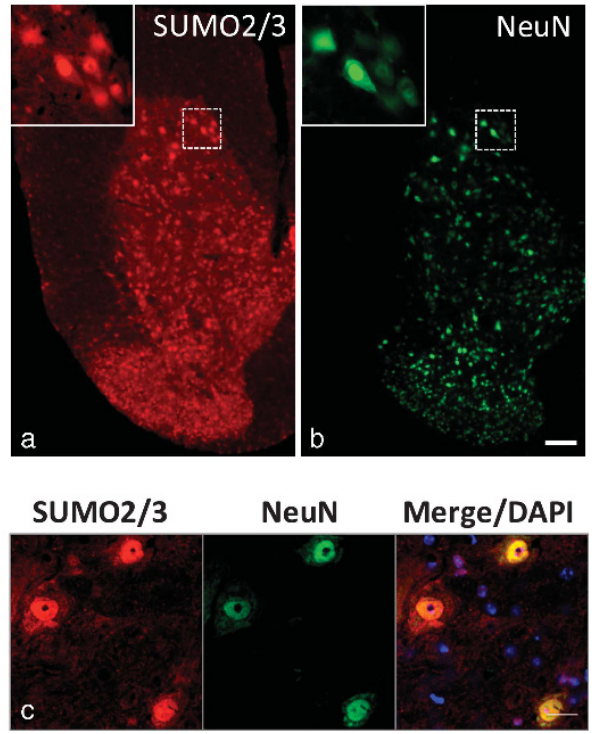

Figure 3 SUMO2/3 immunoreactivity is found in spinal cord neurons, and a massive nuclear accumulation of SUMO2/3-conjugated proteins occurred after transient spinal cord ischemia. Representative immunofluorescence staining depicts the pattern of SUMO2/3 conjugation in control spinal cord lumbar segments (A) and ischemic (B) lumbar segments of a mouse subjected to $10 \mathrm{~min}$ of ischemia and $6 \mathrm{~h}$ of reperfusion. At the end of the experiment, the spinal cord was perfusion fixed, and tissue sections were processed for immunofluorescence staining as described in detail in Materials and methods. NeuN staining and 4,6-diamidino-2-phenylindole (DAPI) (blue) were used to identify neurons and nuclei, respectively. Spinal cord overview ((a) SUMO2/3; (b) NeuN; scale bar: $200 \mu \mathrm{m}$ ) with magnification (insets; ventral horn). High magnification (c) derived from confocal microscopy (scale bar: $20 \mu \mathrm{m}$ ).

NeuN. In control spinal cord segments not affected by ischemia (Figure 3A), SUMO2/3 immunoreactivity was confined to the cytoplasm of neurons. In spinal cord lumbar segments exposed to transient ischemia, strong SUMO2/3 immunoreactivity was present in the nuclei and, to a much less extent, in the cytoplasm of postischemic neurons (Figure 3B). This suggests massive post-ischemic nuclear accumulation of SUMO2/3-conjugated proteins.

\section{DISCUSSION}

Spinal cord ischemia is the most debilitating complication associated with thoracoabdominal aortic aneurysm repair. ${ }^{1}$ Here, we used a recently developed experimental model of spinal cord ischemia to investigate ischemia-induced changes in levels of SUMO1- and SUMO2/3-conjugated proteins. Most of the experimental spinal cord ischemia studies published so far have been performed on rabbits or rats. Using a mouse model provides the significant advantage that identified mechanisms of injury or protection from injury can be verified in the respective genetically modified mice. The stress responses triggered by a transient interruption of spinal cord blood supply in rabbits and rats ${ }^{12-14}$ are very similar to those we observed in our new mouse model (Figure 1). This suggests that our new model is reliable and that stress responses are not species dependent.

To the best of our knowledge, this is the first study that investigates global SUMO conjugation in the spinal cord. We found strong SUMO1 and SUMO2/3 immunoreactivity in neurons (Figure 3, and data not shown). In spinal cord astrocytes of the SOD1-G93A mouse model of amyotrophic lateral sclerosis, the EAAT2 astroglial glutamate transporter is cleaved by caspase-3, generating a proteolytic fragment that is SUMO conjugated. ${ }^{15}$ This suggests that the SUMO conjugation machinery is present in spinal cord astrocytes but most probably at a much lower activity level than in neurons.
We found distinct differences in the pattern of SUMO1 and SUMO2/3 immunoreactivity in control and post-ischemic spinal cord neurons. Whereas SUMO1 immunoreactivity did not change after ischemia as demonstrated by western blot analysis (Figure 2) and immunofluorescence staining (data not shown), transient ischemia triggered massive activation of SUMO2/3 conjugation (Figure 2) and markedly changed the pattern of SUMO2/3 immunoreactivity from a predominantly cytoplasmic staining in control neurons to strong nuclear staining in post-ischemic neurons (Figure 3).

Many of the SUMO target proteins identified so far are nuclear proteins involved in gene expression and genome stability. ${ }^{5,6}$ SUMOylation is involved in many of the processes activated in the post-ischemic spinal cord. These include DNA damage repair, ${ }^{16,17}$ ubiquitin conjugation and proteasomal degradation of proteins, ${ }^{18,19}$ NF- $\kappa \mathrm{B}$ activation, ${ }^{20,21}$ hypoxia-inducible factor activation ${ }^{22,23}$ and oxidative stress. ${ }^{24,25}$ Therefore, the post-ischemic activation of SUMO2/3 conjugation is highly likely to have a major impact on the fate of post-ischemic neurons. Furthermore, several SUMO target proteins have important acute effects on neuronal functions. ${ }^{26}$ Such proteins include the mRNA-binding protein $\mathrm{La}^{27}$ the kainate receptor subunit GluR6 ${ }^{28}$ and the potassium channel Kv1.5, ${ }^{29}$ suggesting a role for SUMO conjugation in ion fluxes and neuronal excitability.

Various cellular stress conditions activate SUMO2/3 conjugation, including metabolic, genotoxic, oxidative and thermal stress, ${ }^{30}$ implying that this post-translational protein modification is a global stress response. Neurons are extremely sensitive to ischemia-like conditions when SUMO2/3 conjugation is blocked by silencing their expression. ${ }^{10}$ This finding provides further evidence that the post-ischemic activation of SUMO2/3 conjugation is a protective stress response that helps cells to better withstand a severe form of stress. Genetically manipulated animals are now required to verify the role of SUMO2/3 conjugation in post-ischemic neurons and to identify the SUMOylated proteins. Transgenic mice over- 
expressing the SUMO-conjugating enzyme Ubc9 are partially protected from ischemia-induced damage. ${ }^{31}$ However, Ubc9 also has SUMO conjugation-independent functions. ${ }^{32}$ Transient ischemia experiments on SUMO knockout animals will provide important information on the role of SUMO paralogues in the recovery of neurons from ischemic stress. SUMO transgenic animals may help us to identify the proteins that are SUMO conjugated after, but not before, ischemia. Such investigations will be integral to unraveling the mechanisms by which SUMO conjugation imparts neuroprotection.

In conclusion, results from this study provide evidence that the SUMO2/3 conjugation pathway is massively activated in postischemic spinal cord neurons in our new mouse model. The SUMO conjugation pathway could then be a promising target for the development of preventive and therapeutic strategies to improve outcomes following an ischemic event in the spinal cord.

\section{DATA ARCHIVING}

There were no data to deposit.

\section{CONFLICT OF INTEREST}

The authors declare no conflict of interest.

\section{ACKNOWLEDGEMENTS}

We are grateful to Dr John M Hallenbeck, National Institutes of Health, for providing the SUMO1 and SUMO2/3 polyclonal antibodies; and to Kathy Gage for her excellent editorial contribution in the preparation of this manuscript. This study was supported by funds provided by the Department of Anesthesiology, Duke University Medical Center.

1 Schepens MA, Heijmen RH, Ranschaert W, Sonker U, Morshuis WJ. Thoracoabdominal aortic aneurysm repair: results of conventional open surgery. Eur J Vasc Endovasc Surg 2009; 37: 640-645.

2 Cambria RP, Davison JK, Carter C, Brewster DC, Chang Y, Clark KA et al. Epidural cooling for spinal cord protection during thoracoabdominal aneurysm repair: a five-year experience. J Vasc Surg 2000; 31: 1093-1102.

3 Safi HJ, Miller 3rd CC, Huynh TT, Estrera AL, Porat EE, Winnerkvist AN et al. Distal aortic perfusion and cerebrospinal fluid drainage for thoracoabdominal and descending thoracic aortic repair: ten years of organ protection. Ann Surg 2003; 238: 372-380.

4 Coselli JS, Lemaire SA, Koksoy C, Schmittling ZC, Curling PE. Cerebrospinal fluid drainage reduces paraplegia after thoracoabdominal aortic aneurysm repair: results of a randomized clinical trial. J Vasc Surg 2002; 35: 631-639.

5 Rosas-Acosta G, Russell WK, Deyrieux A, Russell DH, Wilson VG. A universal strategy for proteomic studies of SUMO and other ubiquitin-like modifiers. Mol Cell Proteomics 2005; 4: 56-72.

6 Bruderer R, Tatham MH, Plechanovova A, Matic I, Garg AK, Hay RT. Purification and identification of endogenous polySUMO conjugates. EMBO Rep 2011; 12: 142-148.

7 Yang W, Sheng H, Warner DS, Paschen W. Transient focal cerebral ischemia induces a dramatic activation of small ubiquitin-like modifier conjugation. J Cereb Blood Flow Metab 2008; 28: 892-896.

8 Yang W, Sheng H, Warner DS, Paschen W. Transient global cerebral ischemia induces a massive increase in protein sumoylation. J Cereb Blood Flow Metab 2008; 28 269-279.
9 Wang Z, Yang W, Britz GW, Lombard FW, Warner DS, Sheng H. Development of a simplified spinal cord ischemia model in mice. J Neurosci Meth 2010; 189: 246-251.

10 Datwyler AL, Lattig-Tunnemann G, Yang W, Paschen W, Lee SL, Dirnagl U et al. SUMO2/3 conjugation is an endogenous neuroprotective mechanism. J Cereb Blood Flow Metab 2011; 31: 2152-2159.

11 Malakhov MP, Mattern MR, Malakhova OA, Drinker M, Weeks SD, Butt TR. SUMO fusions and SUMO-specific protease for efficient expression and purification of proteins. J Struct Funct Genomics 2004; 5: 75-86.

12 Penas C, Guzman MS, Verdu E, Fores J, Navarro X, Casas C. Spinal cord injury induces endoplasmic reticulum stress with different cell-type dependent response. J Neurochem 2007; 102: 1242-1255.

$13 \mathrm{Lu} \mathrm{K}$, Cho CL, Liang CL, Chen SD, Liliang PC, Wang SY et al. Inhibition of the MEK/ERK pathway reduces microglial activation and interleukin-1-beta expression in spinal cord ischemia/reperfusion injury in rats. J Thorac Cardiovasc Surg 2007; 133: 934-941.

14 Sakurai M, Nagata T, Abe K, Horinouchi T, Itoyama Y, Tabayashi K. Survival and death-promoting events after transient spinal cord ischemia in rabbits: induction of Akt and caspase3 in motor neurons. J Thorac Cardiovasc Surg 2003; 125: 370-377.

15 Foran E, Bogush A, Goffredo M, Roncaglia P, Gustincich S, Pasinelli P et al. Motor neuron impairment mediated by a sumoylated fragment of the glial glutamate transporter EAAT2. Glia 2011; 59: 1719-1731.

16 Bergink S, Jentsch S. Principles of ubiquitin and SUMO modifications in DNA repair. Nature 2009; 458: 461-467.

17 Lin R, Roseborough G, Dong Y, Williams GM, Wei C. DNA damage and repair system in spinal cord ischemia. J Vasc Surg 2003; 37: 847-858.

18 Tatham MH, Matic I, Mann M, Hay RT. Comparative proteomic analysis identifies a role for SUMO in protein quality control. Sci Signal 2011; 4: rs4.

19 Yamauchi T, Sakurai M, Abe K, Matsumiya G, Sawa Y. Ubiquitin-mediated stress response in the spinal cord after transient ischemia. Stroke 2008; 39: 1883-1889.

20 Fan L, Wang K, Shi Z, Die J, Wang C, Dang X. Tetramethylpyrazine protects spinal cord and reduces inflammation in a rat model of spinal cord ischemia-reperfusion injury. J Vasc Surg 2011; 54: 192-200.

21 Lee MH, Miyamoto S. Expanding NFkappaB and SUMO ties. Cell Cycle 2011; 10: 3983-3984.

22 Xiaowei H, Ninghui Z, Wei X, Yiping T, Linfeng X. The experimental study of hypoxiainducible factor- 1 alpha and its target genes in spinal cord injury. Spinal Cord 2006; 44: 35-43.

23 Bae SH, Jeong JW, Park JA, Kim SH, Bae MK, Choi SJ et al. Sumoylation increases HIF-1alpha stability and its transcriptional activity. Biochem Biophys Res Commun 2004; 324: 394-400.

24 Grant MM. Identification of SUMOylated proteins in neuroblastoma cells after treatment with hydrogen peroxide or ascorbate. BMB Rep 2010; 43: 720-725.

25 Chronidou F, Apostolakis E, Papapostolou I, Grintzalis K, Georgiou CD, Koletsis EN et al. Beneficial effect of the oxygen free radical scavenger amifostine (WR-2721) on spinal cord ischemia/reperfusion injury in rabbits. J Cardiothorac Surg 2009; 4: 50.

26 Wilkinson KA, Nakamura Y, Henley JM. Targets and consequences of protein SUMOylation in neurons. Brain Res Rev 2010; 64: 195-212.

27 van Niekerk EA, Willis DE, Chang JH, Reumann K, Heise T, Twiss JL. Sumoylation in axons triggers retrograde transport of the RNA-binding protein La. Proc Natl Acad Sci USA 2007; 104: 12913-12918.

28 Martin S, Nishimune A, Mellor JR, Henley JM. SUMOylation regulates kainatereceptor-mediated synaptic transmission. Nature 2007; 447: 321-325.

29 Benson MD, Li QJ, Kieckhafer K, Dudek D, Whorton MR, Sunahara RK et al. SUMO modification regulates inactivation of the voltage-gated potassium channel Kv1.5. Proc Natl Acad Sci USA 2007; 104: 1805-1810.

30 Yang W, Sheng H, Homi HM, Warner DS, Paschen W. Cerebral ischemia/stroke and small ubiquitin-like modifier (SUMO) conjugation-a new target for therapeutic intervention? J Neurochem 2008; 106: 989-999.

31 Lee YJ, Mou Y, Maric D, Klimanis D, Auh S, Hallenbeck JM. Elevated global SUMOylation in Ubc9 transgenic mice protects their brains against focal cerebral ischemic damage. PLoS One 2011; 6: e25852.

32 Zhu S, Sachdeva M, Wu F, Lu Z, Mo YY. Ubc9 promotes breast cell invasion and metastasis in a sumoylation-independent manner. Oncogene 2010; 29: 1763-1772. 